\title{
The Dark Side of Seizure: Case Series of a T2 Dark- Through Pattern of Peri-ictal Diffusion Restriction
}

Eleanor $\mathrm{Chu}^{1^{*}}$, Jennifer Soun ${ }^{1}$ and Jason Handwerker ${ }^{2}$

${ }^{1}$ University of California Irvine, Irvine, California, USA

${ }^{2}$ University of California San Diego, San Diego, California, USA

Correspondence to:

Dr. Eleanor Chu, MD

UC Irvine Medical Center

101 The City Drive South Building 20

Room 144, Orange, CA 92868, United States

Tel: (415) 420-7381

Fax: (714) 456-6832

E-mail: eleanor.chu@gmail.com

Received: June 23, 2020

Accepted: July 06, 2020

Published: July 07, 2020

Citation: Chu E, Soun J, Handwerker J. 2020. The Dark Side of Seizure: Case Series of a T2 Dark-Through Pattern of Peri-ictal Diffusion Restriction. J Neuroimaging Psychiatry Neurol 5(2): 20-23.

Copyright: (c) $2020 \mathrm{Chu}$ et al. This is an Open Access article distributed under the terms of the Creative Commons Attribution 4.0 International License (CC-BY) (http://creativecommons. org/licenses/by/4.0/) which permits commercial use, including reproduction, adaptation, and distribution of the article provided the original author and source are credited.

Published by United Scientific Group

\begin{abstract}
Analogous to T2 shine-through, T2 hypointensity can "dark-through" on diffusion weighted imaging (DWI) and mask diffusion restriction. In such cases, diffusion restriction is evident only by apparent diffusion coefficient (ADC) hypointensity, which is often subtle and easily missed without corresponding DWI hyperintensity. Because diffusion restriction may be present in the setting of seizure, avoiding this pitfall can aid in seizure diagnosis for patients without any other magnetic resonance imaging (MRI) findings. We present a case series of nine patients in the peri-ictal period with T2 dark-through on MRI to raise awareness of this finding and its potential clinical role.
\end{abstract}

\section{Keywords}

T2 hypointensity, Diffusion restriction, Seizure

\section{Introduction}

T2 dark-through describes diffusion restriction in the setting of T2 hypointensity.Just as T2 hyperintensity can "shine-through" on diffusion weighted imaging (DWI), T2 hypointensity can "dark-though" on DWI. DWI signal is influenced by multiple factors including T2 signal, which is demonstrated in the equation: DWI signal intensity $=k P D e^{-T E / T 2} e^{-b A D C}$, where $\mathrm{k}$ is a constant, $\mathrm{PD}$ is the proton density, TE is the echo time, and $\mathrm{b}$ is the $\mathrm{b}$-value [1]. Understanding T2 dark-though is essential for detecting diffusion restriction of T2 hypointense processes where subtle apparent diffusion coefficient (ADC) hypointensity may be missed without corresponding DWI hyperintensity.

There have been reports of $\mathrm{T} 2$ dark-through in patients with seizure, hyperglycemia, Moma-Moya, Sturge-Weber, intracranial neoplasm, intracranial infection, and trauma [1-10]. We present a case series of nine patients with periictal T2 dark-through, including the first documented cases in the setting of venous thrombosis and subdural hemorrhage.

\section{Cases of Hyperglycemia}

A 6-year-old male presented with 3 episodes of focal right-sided shaking and severe hyperglycemia $(414 \mathrm{mg} / \mathrm{dL})$. MRI showed left parietal T2 dark-through (Figure 1). Patient improved following the treatment of hyperglycemia.

A 52-year-old female presented with episodic confusion over the course 


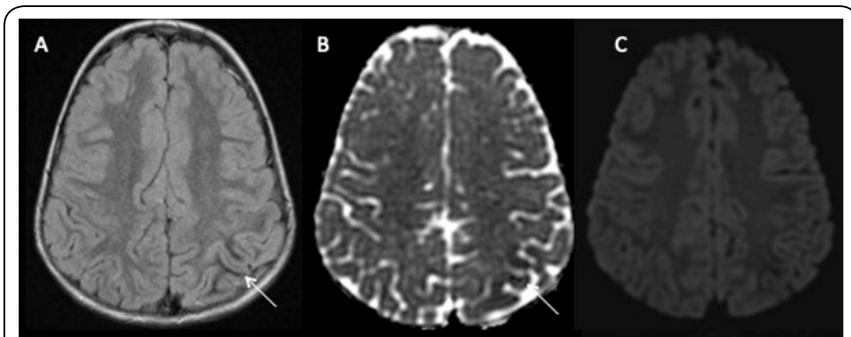

Figure 1: MRI shows left parietal subcortical hypointensity on (A) FLAIR and (B) ADC without corresponding hyperintensity on (C) DWI.

of 6 months and severe hyperglycemia (413 mg/dL). MRI showed right parietal T2 dark-through (Figure 2). Electroencephalogram (EEG) showed right intermittent rhythmic delta activity and background slowing. Patient improved following the treatment of hyperglycemia.

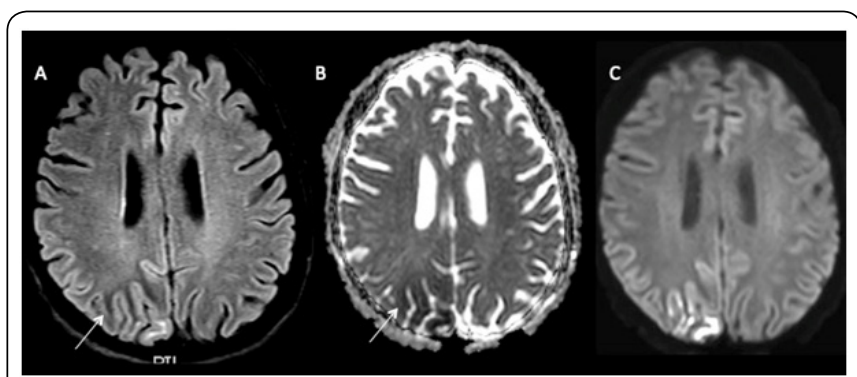

Figure 2: MRI shows right parietal subcortical hypointensity on (A) FLAIR and (B) ADC without corresponding hyperintensity on (C) DWI. There is a separate adjacent area of cortical diffusion restriction with hypointensity on (B) ADC and hyperintensity on (C) DWI.

A 56-year-old male presented with episodic left lower quadrant visual changes 50 times per day and severe hyperglycemia $(451 \mathrm{mg} / \mathrm{dL})$. Initial MRI showed right parietal T2 dark-through (Figure 3). Patient improved following the treatment of hyperglycemia. Findings were less prominent on 3-month follow-up MRI.

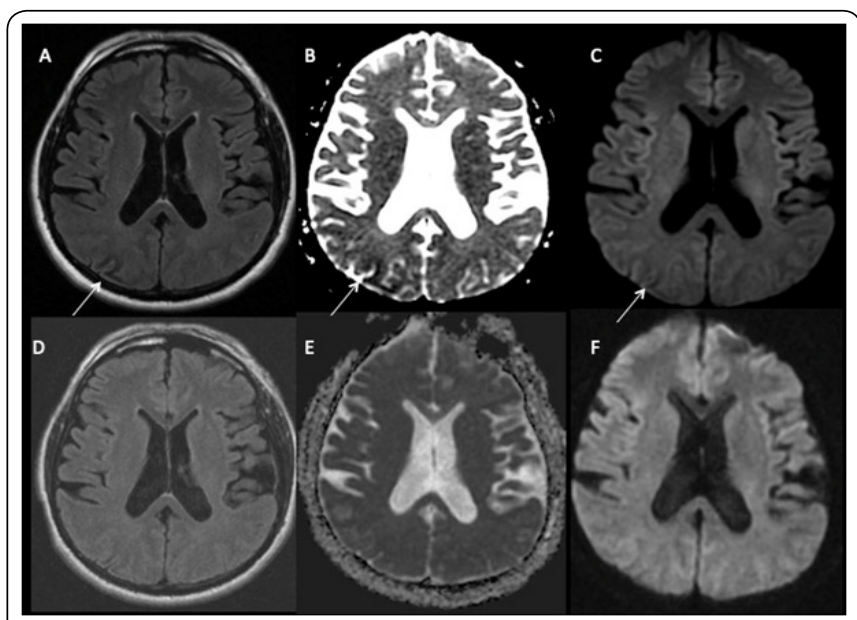

Figure 3: Initial MRI shows right parietal subcortical hypointensity on (A) FLAIR, (B) ADC, and (C) DWI. 3-month follow-up MRI shows less prominent findings on (D) FLAIR, (E) ADC, and (F) DWI.

\section{Case of Venous Thrombosis}

A 32-year-old male presented with generalized tonic- clonic convulsions lasting 1 minute and superior sagittal sinus thrombosis. MRI showed left parietal T2 dark-through, venous thrombosis, and leptomeningeal enhancement, the latter thought to be related to venous stasis (Figure 4). Patient was diagnosed with protein $\mathrm{C}$ deficiency and improved following anticoagulation.

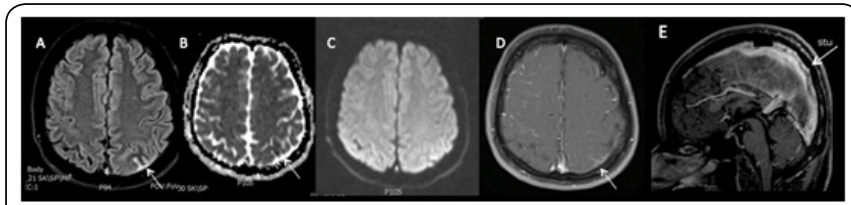

Figure 4: MRI shows left parietal subcortical hypointensity on (A) FLAIR and (B) ADC without corresponding hyperintensity on (D) DWI, sulcal hyperintensity on (A) FLAIR, leptomeningeal enhancement on (D) axial $\mathrm{T} 1+$, and thrombus in the superior sagittal sinus on $(\mathrm{E})$ sagittal $\mathrm{T} 1+$.

\section{Case of Hyperglycemia and Venous Thrombosis}

A 62 year-old-female presented with left upper extremity coordination difficulties over the course of 1 week with 2 episodes of shaking lasting 2-3 minutes, severe hyperglycemia $(505 \mathrm{mg} / \mathrm{dL})$, and right transverse sinus thrombosis.Initial MRI showed right parietal T2 dark-through, venous thrombosis, and leptomeningeal enhancement, the latter thought to be related to venous stasis (Figure 5). Patient improved following the treatment of hyperglycemia and anticoagulation. T2 darkthrough was resolved on 1-month follow-up MRI.

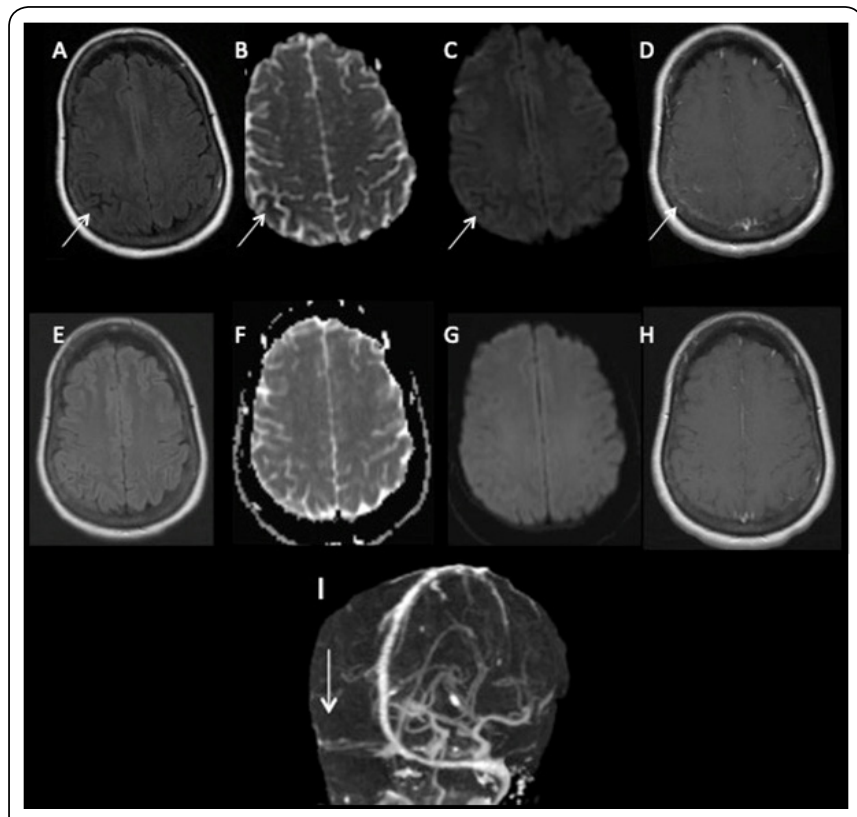

Figure 5: Initial MRI shows right parietal subcortical hypointensity on (A) FLAIR, (B) ADC, and (C) DWI, leptomeningeal enhancement on (D) T1+, and right transverse sinus thrombosis on (I) MRV. 1-month followup MRI shows resolution of findings on (E) FLAIR, (F) ADC, (G) DWI, and $(\mathrm{H}) \mathrm{T} 1+$. Follow-up MRV was not available.

\section{Cases of Meningitis}

A 9-year-old female presented with right upper extremity shaking and numbness in the setting of meningitis. Cerebrospinal fluid analysis showed an opening pressure 
of $24 \mathrm{~cm} \mathrm{H} 2 \mathrm{O}, 93 \mathrm{WBC}$ per uL (48\% neutrophils, 45\% lymphocytes), $26.5 \mathrm{mg} / \mathrm{dL}$ of protein, $60 \mathrm{mg} / \mathrm{dL}$ of glucose, and negative gram stain. MRI demonstrated left frontal T2 dark-through and leptomeningeal enhancement (Figure 6). Patient was lost to follow-up.

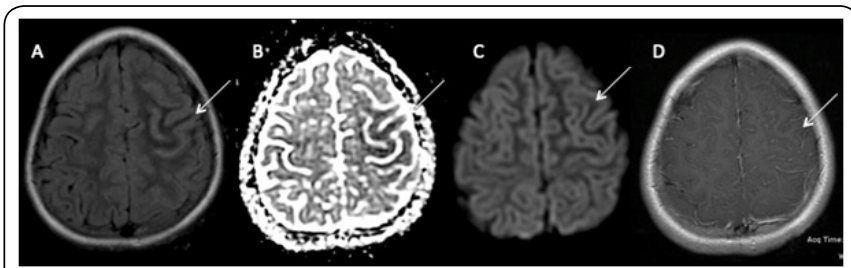

Figure 6: MRI shows left frontal subcortical hypointensity on (A) FLAIR, (B) ADC, and (C) DWI, and leptomeningeal enhancement on (D) T1+.

A 16-year-old male presented with left upper extremity and left facial twitching for 2 minutes in the setting of meningitis. MRI demonstrated right frontal T2 darkthrough, sulcal FLAIR hyperintensity, and leptomeningeal enhancement (Figure 7). Patient was lost to follow-up.

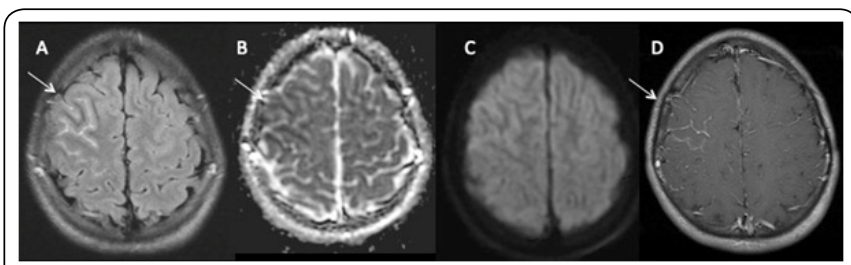

Figure 7: MRI shows right frontal subcortical hypointensity on (A) FLAIR and (B) ADC without corresponding hyperintensity on (C) DWI, sulcal hyperintensity on (A) FLAIR, and leptomeningeal enhancement on (D) $\mathrm{T} 1+$

\section{Case of Sturge-Weber}

A 2-year-old male with history of Sturge-Weber presented with seizure of unspecified semiology. MRI demonstrated right cerebral T2 dark-through and stigmata of Sturge-Weber including asymmetric smaller right cerebral hemisphere with pial angiomatosis (Figure 8). CT showed subcortical calcification only in the posterior right cerebral hemisphere, despite diffuse $\mathrm{T} 2$ dark-through throughout the right cerebral

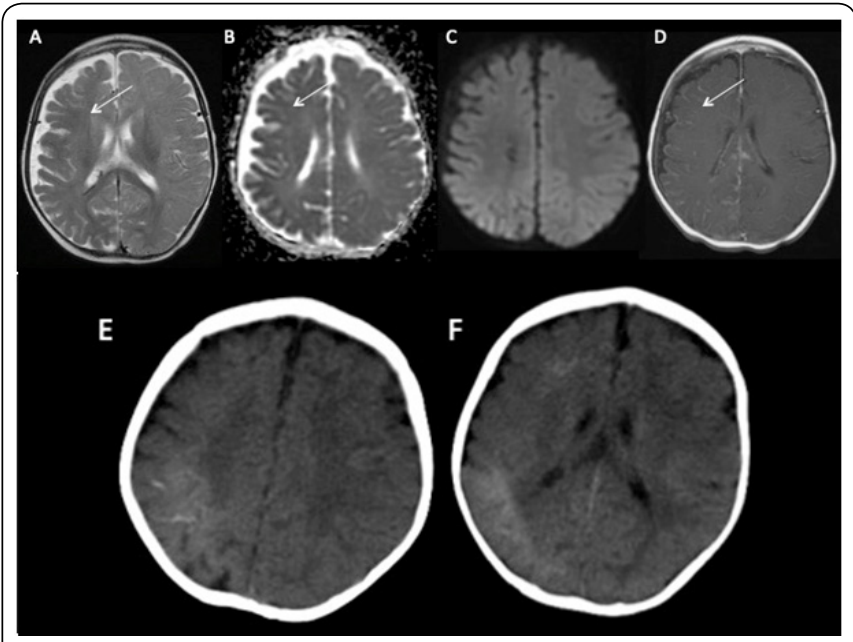

Figure 8: MRI shows diffuse right cerebral hypointensity on (A) T2 and (B) ADC without corresponding hyperintensity on (C) DWI. Stigmata of Sturge-Weber include asymmetric smaller right cerebral hemisphere with pial angiomatosis on (D) T1+ and calcification on (E) and (F) CT. hemisphere. The patient underwent right hemispherectomy due to uncontrolled seizure.

\section{Case of Subdural Hematoma}

A 78-year-old female presented with one episode of right upper extremity tonic-clonic movement and intermittent right-sided weakness lasting 1-5 minutes followed by sleepiness in the setting of subdural hemorrhage. MRI showed left frontal T2 dark-through (Figure 9). The patient improved following hematoma evacuation and seizure management.

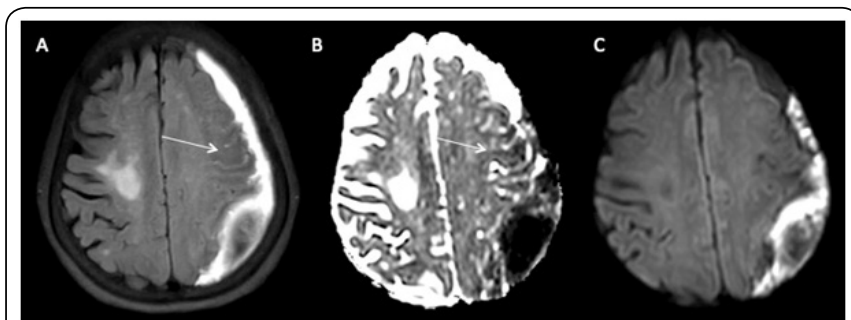

Figure 9: MRI shows left frontal subcortical hypointensity on (A) FLAIR and (B) ADC without corresponding hyperintensity on (C) DWI and a left subdural hematoma.

\section{Discussion}

T2 dark-through is an under-recognized pattern of diffusion restriction where T2 hypointensity "darksthrough" on DWI and masks signal changes. When this happens, restricted diffusion can only be identified by $\mathrm{ADC}$ hypointensity, which may be subtle and easily missed. We present a case series of peri-ictal T2 dark-through, including the first documented cases in the setting of venous thrombosis and subdural hemorrhage, to raise awareness of this finding and its potential clinical role.

The T2 hypointensity component of T2 dark-through has been attributed to the susceptibility effects of paramagnetic substances like deoxyhemoglobin and free radicals [1]. These materials can accumulate from (1) oxygen demand exceeding oxygen supply despite compensatory increased blood flow, such as in the setting of seizure and super-imposed stressors, or (2) insufficient venous drainage, such as in Sturge-Weber, venous thrombosis, or mass effect from adjacent subdural hemorrhage. In the case of Sturge-Weber, axonal hypermyelination and other structural white matter abnormalities have also been proposed as mechanisms for T2 hypointensity [6].

The ADC hypointensity component of T2 dark-through may be related to a combination of hypoxia and excitatory neurotransmitters leading to intracellular edema. In the setting of hypoxia, sodium/potassium-ATPase pump failure results in intracellular shift of sodium and water [11]. Excitatory neurotransmitters released during seizure activate ion channel coupled receptors, which allow the influx of sodium and water and can activate the cell death cascade $[12,13]$. Although the precise combination and role of these mechanisms are still unclear, it is believed that intracellular edema stemming from hypoxia and over-excitation in the peri-ictal state may be responsible for diffusion restriction. 
Patients that received follow-up imaging did not have evidence of encephalomalacia. Plausible explanations for this include: (1) T2 dark-through is reversible if underlying seizure and stressors are treated promptly; (2) T2 dark-through may cause mild tissue injury evident only on histology; (3) our follow-up imaging did not allow sufficient time for the full extent of the T2 dark-through event to manifest. Although imaging was obtained within 24 hours of seizure, the precise time-course is unknown. It is possible many cases of T2 darkthrough are resolved by the time patients are scanned. Prompt imaging in the acute setting and long-term follow-up imaging may increase the sensitivity for T2 dark-through and more reliably define its time course and reversibility.

Although there is a correlation between T2 dark-through and seizure, a relationship of causality is yet to be determined. Regardless, recognizing T2 dark-through may have important implications for seizure localization and managing seizures with potentially reversible underlying etiologies.

Our case series is limited by retrospective review of data, small sample size, and qualitative image analysis. Future prospective studies with a larger sample size and quantitative T2 dark-through analysis can determine the ADC and T2 thresholds for certain clinical outcomes, and better characterize the patient population who will exhibit T2 dark-through and benefit from a more comprehensive workup for comorbidities.

\section{Conclusion}

T2 dark-through is an under-recognized presentation of diffusion restriction occurring in the peri-ictal period. This finding should prompt the radiologist to look for superimposed stressors such as metabolic and vascular abnormalities. Although there is a correlation between T2 dark-through and seizure, a relationship of causality is yet to be determined. With further study, T2 dark-through may have a clinical role in identifying and managing seizure.

\section{Conflict of Interest}

The authors declare no conflict of interest.

\section{References}

1. Hiwatashi A, Kinoshita T, Moritani T, Wang HZ, Shrier DA, et al. 2003. Hypointensity on diffusion-weighted MRI of the brain related to T2 shortening and susceptibility effects. AJR Am J Roentgenol 181(6): 1705-1709. https://doi.org/10.2214/ajr.181.6.1811705

2. Raghavendra S, Ashalatha R, Thomas SV, Kesavadas C. 2007. Focal neuronal loss, reversible subcortical focal T2 hypointensity in seizures with a nonketotic hyperglycemic hyperosmolar state. Neuroradiology 49(4): 299-305. https://doi.org/10.1007/s00234-006-0189-6

3. Lee EJ, Kim KK, Lee EK, Lee JE. 2017. Characteristic MRI findings in hyperglycaemia-induced seizures: diagnostic value of contrastenhanced fluid-attenuated inversion recovery imaging. Clin Radiol 71(12): 1240-1247. https://doi.org/10.1016/j.crad.2016.05.006

4. Urbach H, Berger B, Solymosi L, Zimmermann J. 2020. "Negative T2 shine through" in patients with hyperglycemia and seizures: a frequently overlooked MRI pattern. Neuroradiology 62(7): 895-899. https://doi. org/10.1007/s00234-020-02408-w

5. Chabbert V, Ranjeva JP, Sevely A, Boetto S, Berry I, et al. 1998. Diffusion- and magnetisation transfer-weighted MRI in childhood moya-moya. Neuroradiology 40(4): 267-271. https://doi.org/10.1007/ s002340050583

6. Moritani T, Kim J, Sato Y, Bonthius D, Smoker WRK. 2008. Abnormal hypermyelination in a neonate with Sturge-Weber syndrome demonstrated on diffusion-tensor imaging. J Magn Reson Imaging 27(3): 617-620. https://doi.org/10.1002/jmri.21248

7. Löbel U, Sedlacik J, Reddick WE, Kocak M, Ji Q et al. 2011. Quantitative diffusion-weighted and dynamic susceptibility-weighted contrast-enhanced perfusion MR imaging analysis of T2 hypointense lesion components in pediatric diffuse intrinsic pontine glioma. AJNR Am J Neuroradiol 32(2): 315-322. https://doi.org/10.3174/ajnr.a2277

8. Lee JH, Na DG, Choi KH, Kim KJ, Ryoo JW, et al. 2002. Subcortical low intensity on MR images of meningitis, viral encephalitis, and leptomeningeal metastasis. AJNR Am J Neuroradiol 23(4): 535-542.

9. Okanishi T, Saito Y, Fujii S, Maegaki Y, Fukuda C, et al. 2007. Low signal intensity and increased anisotropy on magnetic resonance imaging in the white matter lesion after head trauma: unrecognized findings of diffuse axonal injury. J Neurol Sci 263(1-2): 218-222. https:// doi.org/10.1016/j.jns.2007.06.048

10. Cerase A, Leonini S, Franceschini R, Grosso S, Venturi C. 2011. Subcortical low-intensity and restricted diffusion after first seizure in a child. J Comput Assist Tomogr 35(4): 501-503. https://doi.org/10.1097/ rct.0b013e31821f5d14

11. Wang Y, Majors A, Najm I, Xue M, Comair Y, et al. 1996. Postictal alteration of sodium content and apparent diffusion coefficient in epileptic rat brain induced by kainic acid. Epilepsia 37(10): 1000-1006. https://doi.org/10.1111/j.1528-1157.1996.tb00539.x

12. Mayer ML, Westbrook GL. 1987. Cellular mechanisms underlying excitotoxicity. Trends Neurosci 10(2): 59-61. https://doi. org/10.1016/0166-2236(87)90023-3

13. Olney JW. 1994. Excitatory transmitter neurotoxicity. Neurobiol Aging 15(2): 259-260. https://doi.org/10.1016/0197-4580(94)90127-9 